\title{
Analysis on Impact of Traffic Accident on Length of Vehicle Queue Based on Cell Transmission Model
}

\author{
Zhijun Zhang \\ School of Mathematics , Baicheng Normal University, Baicheng (13700), \\ Jilin \\ zhangzhijun2929@163.com
}

\begin{abstract}
Traffic parameters including vehicle flow and vehicle speed can be acquired through video recording-manual acquisition method. In this paper, fundamental principle of cell transmission model is introduced, which should be established based on the relationship between length of vehicle queue and actual traffic capacity of the cross section on which a traffic accident occurs, duration of the accident and vehicle flow on upstream road section, based on which EXCEL can be used for processing and obtaining an equinumerous graph for length of vehicle queue on corresponding section. Such equinumerous graph is a dynamic model changing with time, which can reflect the relationship between length of vehicle queue and three other variables. Finally, MATLAB can be used to establish a graph of spatial relationship between length of vehicle queue and three other variables.

Keywords: Traffic Accident; Length of Vehicle Queue; Cell Transmission Model; MATLAB

\section{Introduction}

With the acceleration of China's urbanization, number of vehicle owners has been growing rapidly, which leads to a rapid increase in road traffic volume. Urban roads are characterized by high traffic density and continuity, for which when one traffic lane is occupied, traffic capacity of all other lanes in the section may also be lowered. Even through such situation lasts a short time, it may lead to vehicle queue and then traffic jam. If not properly handled, such situation may even cause regional congestion. Traffic congestion has become an urgent problem to be solved by many countries around the world [1].
\end{abstract}

\section{Investigation Method}

In consideration of number of traffic investigators, restrictions in conditions of investigation and repeated utilization of data acquired through such investigation, video recording-manual acquisition method is used in this paper[2]. A camera is 
installed at a certain high point in place of investigation and camera lens is adjusted to target on location of investigation and begins to shoot continuously. Cross section for investigation preset before shooting is properly marked, and screen shots of the video are indicated in Figure 1. Traffic parameters including vehicle flow and vehicle speed can be obtained through later manual processing of the video.

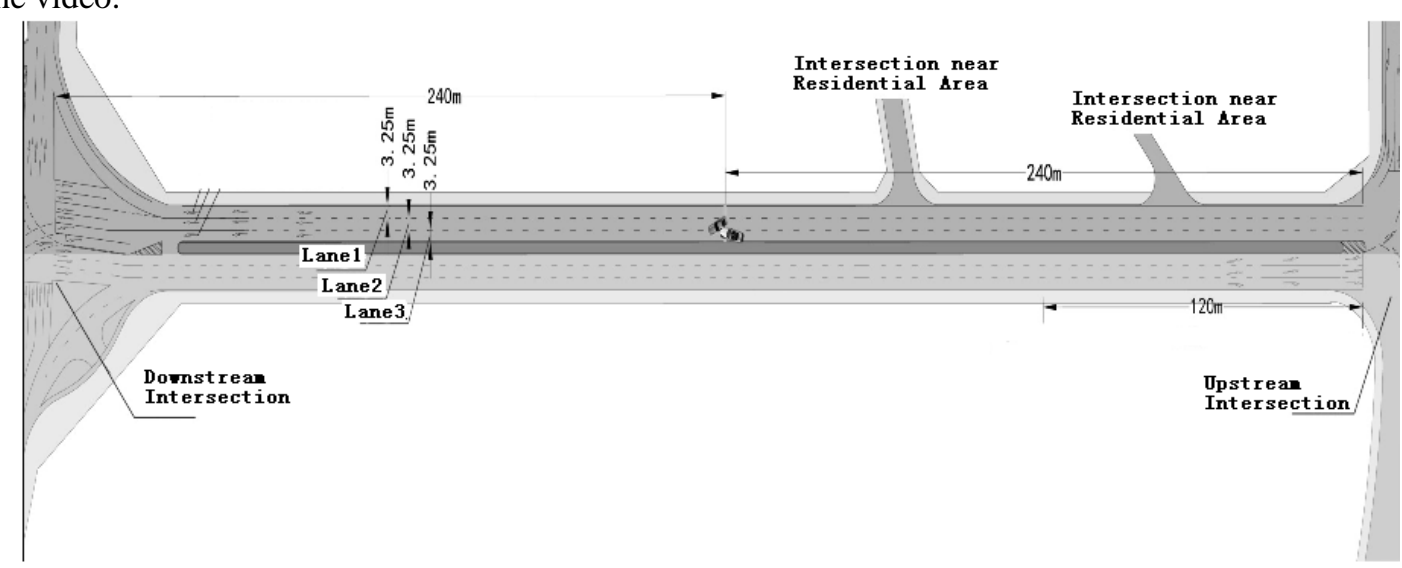

Figure 1 Schematic Diagram of Location of Traffic Accident in Video 1

\section{Principle of Cell Transmission Modeling}

As for Cell Transmission Model (CTM)[3][4], finite differentiability method is used to simplify the relationship between traffic flow and density. CTM is a macro model, in which a certain number of vehicles are viewed as a whole based on traffic condition in a traffic environment and thus observed and verified for convenience. The relationship between traffic flow and density is assumed to be subject to a function in triangular form, as indicated in Figure 2.

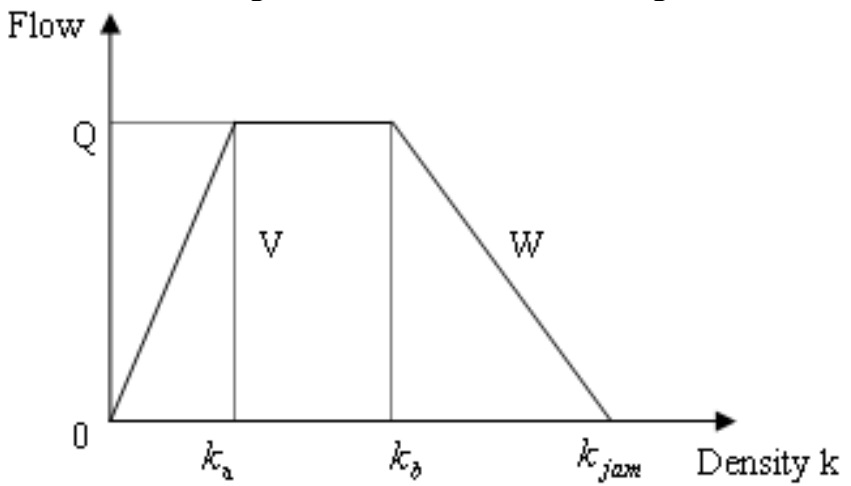

Figure 2 Diagram of Flow-Density Relationship in CTM

Core content of CTM: Road network is a collection of cells with the same length, and length of each cell equals the moving distance of free-flowing 
vehicles within a unit time. If there is no congestion, a vehicle can move from one cell to the next cell within a unit time. Figure 3 indicates the diagram of traffic flow passing through cells in a CTM, in which roads are divided into equal cells. These units are continuously marked from upstream section of roads (from $\mathrm{i}=1$ to $\mathrm{I}$ ).

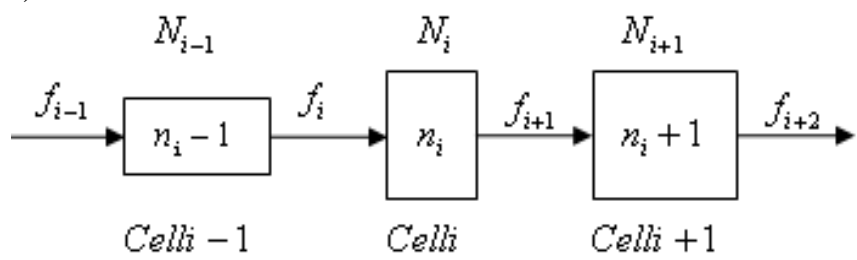

Figure 3 Diagram of Moving of Traffic Flow in CTM

Therefore, formula for volume of each cell (i.e. the maximum allowable number of vehicles contained in each cell (Ni) is indicated in Formula (1). As for road sections of the same nature, volume (Ni) of each cell (i) is determined by number of lanes contained in it because of the same length of each cell. Within the period from $t$ to $t+1$, the allowable number of vehicles $(\mathrm{Q})$ flowing into a cell (i) is the minimum traffic capacity of Cell (i) and Cell (i-1), as indicated in Formula (2). These constants vary with time. For example, in case of a traffic event lasting a short time, $\mathrm{Ni}$ and Qi will both vary with time.

$$
N_{\mathrm{i}}=k_{\text {jam }} \cdot n_{l} \cdot L
$$

(1)

$$
Q_{i}(t)=\min \left\{\begin{array}{c}
\text { Capacity }(i-1) \\
\operatorname{Capacity}(i)
\end{array}\right.
$$

In the formula: $i$ is Cell (i); $i+1$ is the downstream cell of Cell (i); $i-1$ is upstream cell of Cell (i);

$\mathrm{N}_{i}(t)$ _Volume of Cell (i), namely the maximum allowable number of vehicles contained in Cell (i);

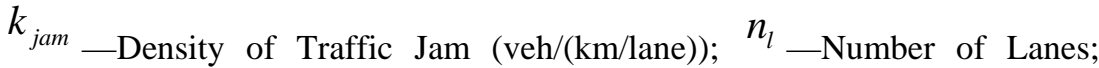
L-Length of Cell $(\mathrm{km})$.

Topological relation of road network when confluence of road sections takes places is indicated in Figure 4, in which BK and CK are transmitting cells and EK is receiving cell. Assume that $p_{\mathrm{k}}$ is the percentage of traffic flow flowing out of Cell (BK); $p_{c k}$ is the percentage of traffic flow flowing out of Cell (CK); and $p_{k}+p_{c k}=1$. If two roads are both under the free-flowing situation, then flow on these two roads is determined by the condition of upstream section, for which $p_{\mathrm{k}=1 \text { and }} p_{c k=0}$. 


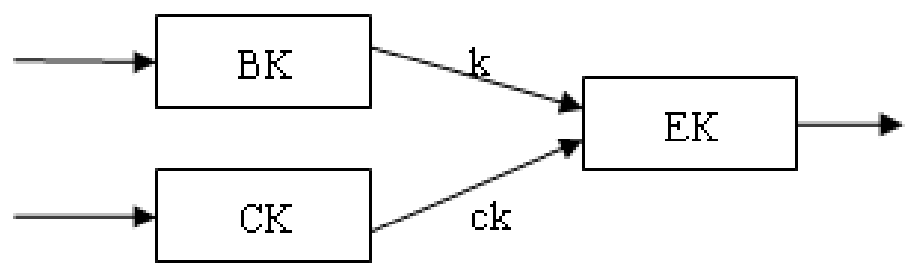

Figure 4 Diagram of Confluence Model of CTM

\section{Modeling for Length of Vehicle Queue and Solution}

With the purpose of actually reflecting the changing process of actual traffic capacity on the cross section in which a traffic accident occurs, road impedance function model is adopted in this paper[5][6], for which mathematical formula is as follows:

$$
\mathrm{c}=\frac{\mathrm{j} q \mathrm{v}(q)}{v_{0}-v(q)}+q
$$

In the formula, $\mathrm{v}(\mathrm{q})$ _Speed of vehicles on a road section when traffic volume is $\mathrm{q}(\mathrm{km} / \mathrm{h}) ; v_{0}$ _Speed of vehicles on a road section in the free-flowing situation $(\mathrm{km} / \mathrm{h})$; C-Actual traffic capacity of a road section (pcu/h); j-Curvature parameter of the model.

$v_{0}$ is the average value of $\mathrm{n}$ speeds of free-flowing vehicles (n data obtained through observation), which can be obtained through $\bar{v}_{S} . \mathrm{V}(\mathrm{q})$ is the average value of speeds of all vehicles passing through within 1 minute when traffic volume is q, which can also be obtained through $\bar{v}_{S}$. Formula of $\bar{v}_{S}$ is as follows:

$$
\bar{v}_{S}=\frac{\sum_{i=1}^{n} v_{i}}{n}
$$

In the formula: $v_{i}$ _ Speed Data (i) collected from the video; n-Number of vehicles observed.

It can be seen though the video that the traffic accident occurs at 16:39:27, and there is an interval of nearly 13 minutes between the above time point and time of evaluation. In this paper, collection of samples from video data is conducted every 30 minutes, so number of collected samples $\mathrm{N}=24$. It can be seen through Figure 1 that the distance between the location of traffic accident and the upstream intersection is $240 \mathrm{~m}$. Length of each cell in this paper is $30 \mathrm{~m}$, so there are 8 cells in total. 
Firstly, 24 values of speed can be collected from the video: $\mathrm{V}=[11.1929 ; 15.2857 ; 11.9429 ; 15.4286 ; 0 ; 0 ; 0 ; 8.1818 ; 6.2528 ; 9.3210 ; 7.5097 ; 0 ; 0 ; 5$. 6250;4.7714;4.7368;3.4615;0;3.2143;0;3.4615;0;0;0]

Speed of free-flowing vehicles can be calculated through Formula (4): $\mathrm{V}_{0}=10.6703$;

Vehicle flow passing through the cross section on which a traffic accident occurs within each 30 seconds can be seen through the video. q=[9;3;12.5;4;12.5;5.5;5.5;2;8;7;6;2;4;3;7.5;3 5;3;4;2;2;2.5;2;1]

Formula of actual traffic capacity of the cross section on which a traffic accident occurs within each 30 seconds can thus be obtained: $\mathrm{C}=\mathrm{q} \times(1+(0.21 \times \mathrm{V}) /(10.6703-\mathrm{V}))$

$\mathrm{C}=[9.0995 ; 3.0332 ; 12.6383 ; 4.0442 ; 12.6383 ; 5.5608 ; 5.5608 ; 2.0221 ; 8.0885 ; 7.07$ $74 ; 6.0664 ; 2.0221 ; 4.0442 ; 3.0332 ; 7.5830 ; 3.0332 ; 5.0553 ; 3.0332 ; 4.0442 ; 2.0221 ; 2$. $0221 ; 2.5277 ; 2.0221 ; 1.0111]$

Equinumerous graph of length of vehicle queue on the road section can be obtained through putting the above measured data into cell transmission model and then conduct processing with EXCEL, as indicated in Figure 5.

\begin{tabular}{|c|c|c|c|c|c|c|c|c|c|}
\hline Time & Cell1 & Cell2 & Cell3 & Cell4 & Cell5 & Cell6 & Cellt & Cell8 & Cel19 \\
\hline $16: 39: 27$ & 0 & 0 & 0 & 3 & 3 & 5 & 7 & 9.09 & 9 \\
\hline $16: 39: 57$ & 13 & 13 & 10 & 8 & 6 & 6 & 3 & 3.03 & 4 \\
\hline $16: 40: 27$ & 0 & 0 & 0 & 6.5 & 8.5 & 10.5 & 12.5 & 12. 64 & 13.5 \\
\hline $16: 40: 57$ & 10 & 11 & 9 & 8 & 6 & 3 & 4 & 4.04 & 3 \\
\hline $16: 41: 27$ & 2 & 1 & 4 & 8.5 & 9.5 & 10.5 & 11.5 & 12.63 & 13.5 \\
\hline $16: 42: 57$ & 12 & 11.5 & 11.5 & 11 & 10 & 6 & 8 & 5.56 & 8.5 \\
\hline $16: 43: 27$ & 2 & 1 & 0 & 2 & 3.5 & 3.5 & 6.5 & 5. 56 & 9.5 \\
\hline $16: 43: 57$ & 6 & 6 & 4 & 6 & 4 & 3 & 4 & 2.02 & 9.5 \\
\hline $16: 44: 27$ & 2 & 2 & 3 & 4 & 4 & 5 & 6 & 8.088 & 8 \\
\hline $16: 44: 57$ & 11 & 11 & 12 & 8 & 6 & 5 & 5 & 7.07 & 7 \\
\hline $16: 45: 27$ & 1 & 0 & 2.5 & 5.5 & 7.5 & 12.5 & 5 & 6.07 & 6 \\
\hline $16: 45: 57$ & 8 & 9 & 9 & 7 & 5 & 4 & 5 & 2. 02 & 8.5 \\
\hline $16: 46: 27$ & 0 & 1 & 1 & 1 & 3 & 5 & 6 & 4. 04 & 7 \\
\hline $16: 46: 57$ & 10 & 10 & 8 & 8 & 7 & 6 & 3 & 3.03 & 6 \\
\hline $16: 47: 27$ & 5 & 5 & 8.5 & 10.5 & 10.5 & 13.5 & 12.5 & 7.58 & 6 \\
\hline $16: 47: 57$ & 13 & 12 & 10 & 9 & 7 & 7 & 4 & 3. 03 & 11 \\
\hline $16: 48: 27$ & 2 & 2 & 4 & 6 & 9 & 10 & 8 & 5.05 & 8 \\
\hline $16: 48: 57$ & 11 & 11 & 7 & 7 & 6 & 4 & 3 & 3.03 & 8 \\
\hline $16: 50: 27$ & 0 & 1 & 5 & 8 & 10 & 14 & 9 & 4. 04 & 6 \\
\hline $16: 50: 57$ & 15 & 14.5 & 10.5 & 8 & 5 & 2 & 1 & 2. 02 & 6 \\
\hline $16: 51: 27$ & 2 & 2 & 7 & 10 & 12.5 & 6.5 & 6 & 2. 02 & 5 \\
\hline $16: 51: 57$ & 14 & 15 & 12.5 & 11.5 & 5.5 & 6 & 5 & 2. 52 & 8 \\
\hline $16: 52: 27$ & 0 & 1 & 3 & 3 & 3 & 6 & 3 & 2. 02 & 7 \\
\hline $16: 52: 57$ & 15 & 15 & 13 & 12 & 9 & 5 & 0 & 1.01 & 7.5 \\
\hline
\end{tabular}

Figure 5 Equinumerous Graph of Length of Vehicle Queue on the Road Section

In Figure 5, Cell (1) corresponds to a location on upstream section; end of Cell (8) is the cross section on which a traffic accident occurs; and length of each cell is $30 \mathrm{~m}$. Vehicle queue occurs at the red mark, and length of such queue equals the length of a cell. Vehicle queue occurs at the gradually changing red mark, length of which equals the length of half a cell. Data inside a cell is the number of vehicles flowing through it observed every 30 seconds. Cell (8) is located at the cross section on which a traffic accident occurs, so data put in Cell (8) indicate the actual traffic capacity on the cross section on which a traffic accident occurs with the purpose of observing the relationship between length of vehicle 
queue and actual traffic capacity in a more obvious way. In addition, vehicles can only move gradually at the same speed along one queue in general situation, for which when degree of freedom of vehicle moving decreases obviously, traffic volume equals or approximately equals traffic capacity of the road section, where traffic volume can be viewed as the actual traffic capacity of the cross section on which a traffic accident occurs.

Figure 5 can be obtained through a cellular model, which is a dynamic model varying with time and can reflect the relationship between length of vehicle queue and three variables including the actual traffic capacity of the cross section on which a traffic accident occurs, duration of the traffic accident and vehicle flow on upstream section in a more obvious way. MATLAB is used to establish the spatial relationship between length of vehicle queue and other variables based on Figure 5, as indicated in Figure 6, Figure 7 and Figure 8.

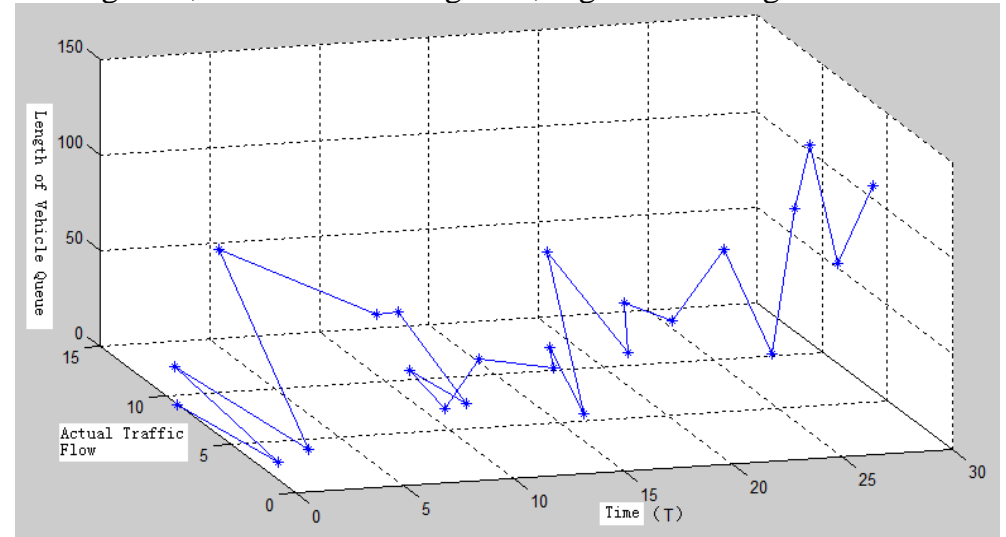

Figure 6 Diagram of Relationship between Length of Vehicle Queue and Actual Traffic Flow Varying with Time

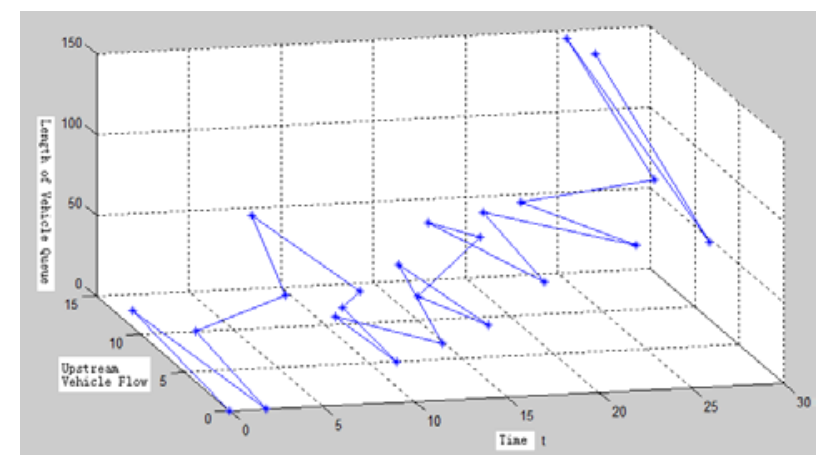

Figure 7 Diagram of Relationship between Length of Vehicle Queue and Upstream Vehicle Flow Varying with Time 


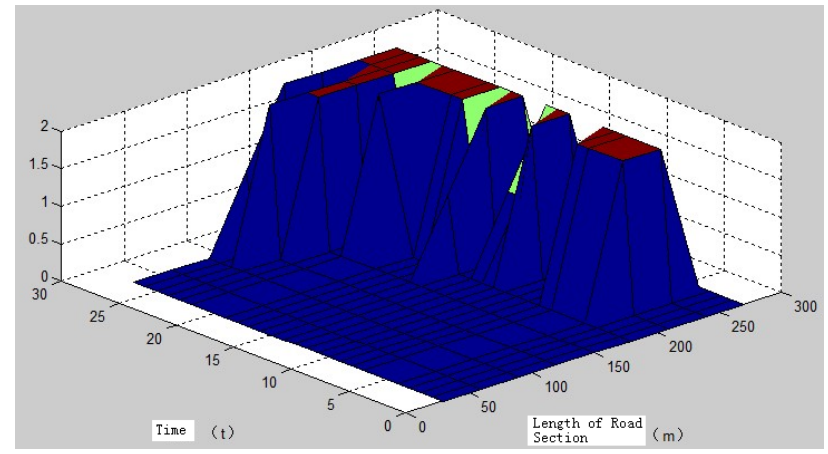

Figure 8 Diagram of Length of Vehicle Queue Varying with Time in Different Road Sections

\section{Conclusion}

In this paper, cell transmission model and road impedance function are used to study the relationship between length of vehicle queue and the actual traffic capacity of the cross section on which a traffic accident occurs, duration of traffic accident and vehicle flow on upstream section when a traffic accident occurs, based on which MATLAB is used to establish the spatial relationship between length of vehicle queue and other variables. The model established in this paper may provide a practical method for urban road occupation for construction work as well as arrangement of roadside parking space and bus stops (except for bus parking bay).

\section{References}

[1] Wang Wenqun and Margaret C. Bell. Summary of Analysis on Duration of Traffic Accidents [J], Journal of Transportation Systems Engineering and Information Technology, 5 (3): 127-130, 2005.

[2]Daganzo C F, The cell trans mission model part II net-work traffic [J], Transportation Research, 29B(2): 79-93, 1995.

[3] Ji Yangbeibei, Du Yuchuan and Sun Lijun. Modeling for Dissipation of Traffic Events Based on Cell Transmission Model [J], Journal of Chongqing Jiaotong University (Natural Science), 27 (3): 442-445, 2008.

[4]Shang Huayan and Huang Haijun. Traffic Information Design Based on Cell Transmission Model [J], Journal of Beijing University of Aeronautics and Astronautics, 34 (2): 234-238, 2008.

[5] Zheng Lili and Peng Guoxiong. Fuzzy Prediction of Delay Caused by Traffic 
Accidents on Highway [J] Journal of Tongji University (Natural Science), 33 (11):1458-146, 2005.

[6] Chen Dewang, Guan Jizhen and Zhu Xueliang. Research on Traffic Flow Parameters Based on Occupation Ratio [J], Journal of Transportation Systems Engineering and Information Technology, 6 (5): 83-86, 2006. 